\title{
A Study of Identical Twins' Palmprints for Personal Authentication
}

\author{
Adams Kong ${ }^{1,2}$, David Zhang ${ }^{2}$, and Guangming $\mathrm{Lu}^{3}$ \\ ${ }^{1}$ Pattern Analysis and Machine Intelligence Lab, University of Waterloo, \\ 200 University Avenue West, Waterloo, Ontario N2L 3G1, Canada \\ adamskong@ieee.org \\ ${ }^{2}$ Biometric Research Centre, Department of Computing, \\ The Hong Kong Polytechnic University, Kowloon, Hong Kong \\ csdzhang@comp.polyu . edu.hk \\ ${ }^{3}$ Biocomputing Research Lab, School of Computer Science and Engineering, \\ Harbin Institute of Technology, Harbin, China \\ csglu@comp.polyu.edu.hk
}

\begin{abstract}
Biometric recognition based on human characteristics for personal identification has attracted great attention. The performance of biometric systems highly depends on the distinctive information in the biometrics. However, identical twins having the closest genetics-based relationship are expected to have maximum similarity between their biometrics. Classifying identical twins is a challenging problem for some automatic biometric systems. In this paper, we summarize the exiting experimental results about identical twins' biometrics including face, iris, fingerprint and voice. Then, we systemically examine identical twins' palmprints. The experimental results show that we can employ lowresolution palmprint images to distinguish identical twins.
\end{abstract}

\section{Introduction}

Biometric systems measuring our biological and behavioral features for personal authentication have inherent advantages over traditional knowledge-based approach such as password and over token-based approach such as physical key. Various biometric systems such as face, iris, retina, fingerprint and signature, were proposed, implemented and deployed in the last thirty years [1]. Biometric systems use the distinctive information in our biometric traits to identify different people. Nevertheless, not all biometrics have sufficient information to classify identical twins having the same genetic expression.

There are two types of twins, monozygotic and dizygotic twins. Dizygotic twins result from different fertilized eggs. Consequently, they have different Deoxyribo Nucleic Acid (DNA). Monozygotic twins, also called identical twins are the result of a single fertilized egg splitting into two individual cells and finally developing into two persons. Thus, identical twins have the same DNA. The frequency of identical twins is about $0.4 \%$ across different populations [2]. Some people believe that this is the limit of face recognition systems [18]. 


\subsection{From DNA to Biometrics}

DNA contains all the genetic information required to generate an organ of a species. The mapping from the genetic information to an organ is very complicated. First of all, the genetic information in DNA molecule is copied to RNA (Ribo Nucleic Acid) molecule. Next, the information in RNA is used to generate amino acids and the amino acids are converted into functioning proteins. The functioning proteins are assembled to be an organ. In this process, genetic information is not the only one factor affecting the organ. It can be influenced by various other factors. As a result, identical twins who share the same genetic expression have many different biometrics including fingerprint, iris and retina $[3,15,17]$. In fact, some biometrics such as faces continually change after we are born. The changes depend on environmental conditions such as living style, diet and climate. They make identical twins more different when they age. Fig. 1 shows two pairs of identical twins at different ages. The older twins in Fig. 1(b) are easier to be distinguished.

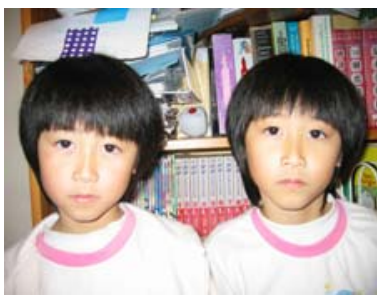

(a)

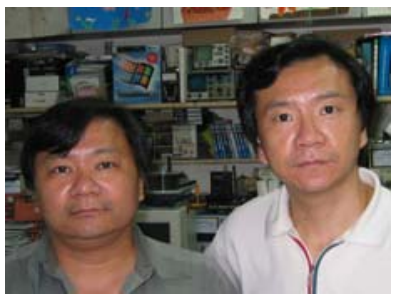

(b)

Fig. 1. Two pairs of identical twins at different ages

\subsection{Motivations}

Identifying identical twins is important for all biometric systems. The systems that cannot handle identical twins have a serious security hole. According to our best knowledge, so far no paper summarizes the testing results of identical twins. In addition, no one investigates the similarity between low-resolution identical twins' palmprints.

The rest of this paper is organized as follows. Section 2 summarizes the testing reports from different sources. Section 3 gives the experimental results of identical twins' palmprints. Section 4 discusses the experimental results and the summary. Finally, Section 5 offers some concluding remarks.

\section{Summary of the Existing Reports}

In this paper, we discuss only the biological features including retina, iris, face, voice and fingerprint that are directly affected by genetic factors. Fig. 2 illustrates identical twins' retinas, irises, fingerprints and palmprints. These images are collected from different pairs of twins. The iris and palmprint images are collected using our selfdesigned devices [20] and the retina images are obtained from [6] with permission to reprint. The fingerprint images are collected using a standard optical fingerprint 
scanner. Fig. 2 shows that the retinas, irises and palmprints can easily be distinguished by human vision. For the fingerprints, we have to pay more attention at the minutiae points, commonly utilized in fingerprint systems. Based on the positions and directions of the minutiae points, the twins' fingerprints can be distinguished without any problem.

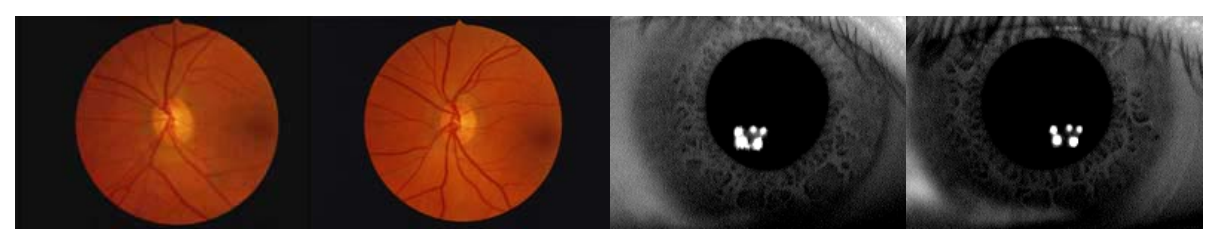

(b)

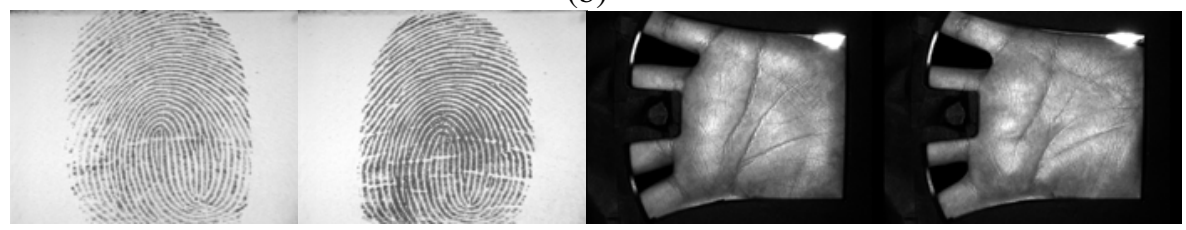

(d)

Fig. 2. Different features from identical twins', (a) retinas, (b) irises, (c) fingerprints and (d) palmprints

In many cases, biometrics are proposed by medical doctors or ophthalmologists [15-16] but almost all the biometric identification systems are designed by engineers. The features discovered by doctors or ophthalmologists and the features applied to authentication systems may not be the same. The iris is a typical example $[6,17]$. Ophthalmologists distinguish irises based on the structural features including moles, freckles, nevi and crypts while current iris recognition systems use binary sequences to represent the textural features. Therefore, the experimental results or observation given by doctors or ophthalmologists about identical twins may not be applicable to automatic biometric systems. In other words, it is essential to test automatic biometric systems on identical twins. Table 1 summarizes the testing results including, iris, face, palmprint and voice. We also give the sizes of testing databases and age ranges of the testing samples in Table 1. The database size refers to the number of different biometrics, not the number of twin pairs. The testing results are represented by the symbols "+" and "-". The symbol "+" denotes that the tested methods can distinguish identical twins, just as normal persons. The symbol "-" denotes that the tested method cannot correctly distinguish them.

All the results in Table 1 are positive, except voice recognition. Some of the results are not significant since their testing databases are too small. Based on [7, 9] and experimental results in Section 3, we ensure that iris, palmprint and fingerprint can be used to separate identical twins. However, testing on large databases is required to verify the results of $3 \mathrm{D}$ face, $2 \mathrm{D}$ face and fusion of lip motion and voice [10-11, 13, 14]. It is generally believed that faces cannot be used for separating identical twins. Experts in National Institute of Standards and Technology (USA) said "although 
identical twins might have slight facial differences, we cannot expect a face biometric system to recognize those differences."[18]. Interestingly, the results in Table 1 contradict our general beliefs.

In addition to fingerprint, palmprint and iris, retina and thermogram are considered as distinctive features for identical twins [9]. So far, we have not obtained any testing report about them.

Table 1. Summary of the existing twin tests

\begin{tabular}{|c|c|c|c|c|}
\hline Biometric & Results & $\begin{array}{c}\text { Age } \\
\text { Ranges }\end{array}$ & $\begin{array}{c}\text { Database } \\
\text { Size }\end{array}$ & $\begin{array}{c}\text { Refer- } \\
\text { ence }\end{array}$ \\
\hline Iris & + & $*$ & $648^{\#}$ & {$[7]$} \\
\hline 3D face & + & $*$ & Several & {$[10-11]$} \\
\hline 2D face & + & $*$ & 20 & {$[13]$} \\
\hline Fingerprint & + & $*$ & 188 & {$[9]$} \\
\hline Palmprint & + & $6-45$ & 106 & Section 3 \\
\hline Voice & - & $*$ & 32 & {$[12]$} \\
\hline Lip motion and speech & + & $18-26$ & 4 & {$[14]$} \\
\hline
\end{tabular}

* The age ranges are not available.

${ }^{\#}$ In this test, 648 right/left iris pairs from 324 persons are tested since our left and right irises are generated from the same DNA.

\section{Study of Twins' Palmprints}

According to our best knowledge, no one studies identical twins' palmprints for automatic personal authentication. In this experiment, we utilize the orientation fields of palmprints as feature vectors to represent low-resolution palmprint images and use angular distance to compare the feature vectors. Readers can refer to [8] for the computational detail of this method. Shorter angular distance represents more similarity between two palmprint images. This method is a modification of our previous work [20]. To compare with the palmprints from general persons and identical twins, we prepare two databases for this study. The details of the databases are given in the following sub-sections.

\subsection{Twin and General Palmprint Databases}

The twin database contains 1028 images collected from 53 pairs of identical twins' palms. We collect the images from their left and right palms. Around 10 images are collected from each palm. All the images are collected by our self-designed palmprint capture device [20]. The image size is $384 \times 284$.

To produce a reliable genuine distribution, we prepare a palmprint database containing 7,752 images from the right and left palms of 193 individuals. This database is called general palmprint database. The images in this database are collected on two separate occasions, two months apart. On each occasion, the subject was asked to provide about 10 images, each of the left palm and the right palm. The average interval between the first and second collections was 69 days. More information about this database can be referred to [20]. 


\subsection{Experimental Results}

To study the similarity between identical twins' palmprints and to obtain twin imposter distribution, we match a palmprint in the twin database with his/her identical twin sibling's palmprints (twin match). We also match every palmprint in the general database with other palmprints in the general database to obtain genuine and imposter (general match) distributions of normal persons. In addition, we match different person's left palmprints and match different person's right palmprints to obtain a side imposter distribution (side match). Total number of genuine matchings, general imposter matchings, side imposter matchings and twin imposter matchings are 74,068, $29,968,808,14,945,448$ and 4,900, respectively. The genuine distribution and imposter distributions of general match, twin match and side match are given in Fig. 3(a). The genuine distribution along with the three imposter distributions in Fig. 3(a) is used to generate the Receiver Operating Characteristics (ROC) curves given in Fig. 3(b). Fig. 3(b) shows that we can use low-resolution palmprint images to distinguish identical twins but identical twins' palms have some inherent correlation, which is not due to side matching.

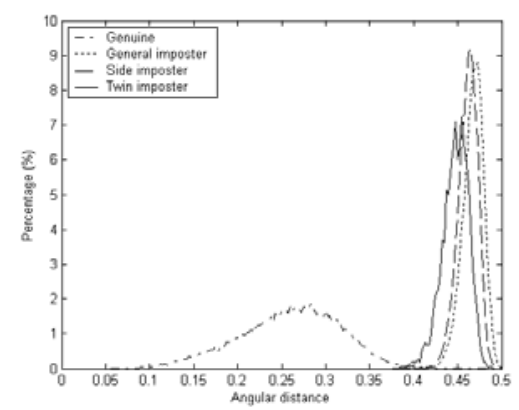

(a)

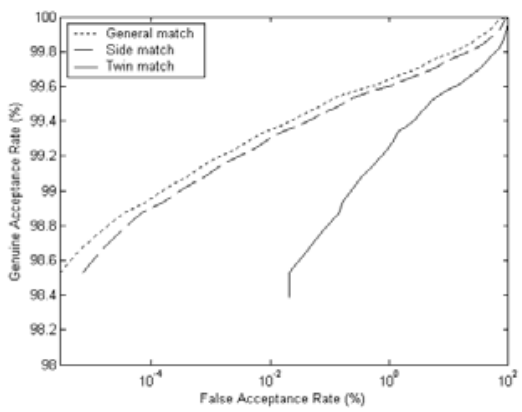

(b)

Fig. 3. Verification results. (a) Twin imposter, side imposter, general imposter and genuine distributions and (b) ROC curves for corresponding distributions.

\section{Discussion}

According to the summary and the experimental results in Section 3, we have confidence to say that, iris, fingerprint and palmprint are three effective biometrics to distinguish identical twins. The subjective comparisons of these three biometrics are given in Table 2. The comments of fingerprint and iris are obtained from [1]. We also agree the comments about palmprint in [1], except collectability. The palmprints discussed in this paper are collected by a CCD camera-based palmprint scanner. Thus, the collectability of palmprint should be similar to that of hand geometry (High). According to Table 2, none of them is perfect. Each of them has strengths and weaknesses. Our low-resolution palmprint recognition method has combined the advantages of hand geometry and fingerprints, with high collectability and high 
Table 2. Comparison of palmprint, fingerprint and iris

\begin{tabular}{|c|c|c|c|}
\hline & Palmprint [8, 20] & Fingerprint [1] & Iris [1] \\
\hline Universality & Middle & Middle & High \\
\hline Distinctiveness & High & High & High \\
\hline Permanence & High & High & High \\
\hline Collectability & High* & Middle & Middle \\
\hline Performance & High & High & High \\
\hline Acceptability & Middle & Middle & Low \\
\hline Circumvention & Middle & Middle & Low \\
\hline
\end{tabular}

* The authors' comments are different from [1].

performance. In addition, low-resolution palmprints do not have the problem of latent prints, which can be used to make artificial fingerprints to fool current commercial fingerprint systems [4].

\section{Conclusion}

In this paper, we have summarized the testing reports about examining biometric systems on identical twins. Although identical twins have the same DNA, their biometric traits including iris palmprints and fingerprint are different. Currently, biometric systems can effectively classify identical twins' irises and fingerprints. The existing reports about face recognition for identical twins give some encouraging results. They show that face is possible to be a foolproof way to tell the differences between identical twins. However, the methods should be tested on larger twin databases. Since their testing databases are too small, their results may not be reliable. In addition to the summary, the experimental results show that identical twins' palmprints are distinguishable but they have some inherent correlation.

\section{References}

1. A.K. Jain, A. Ross and S. Prabhakar, "An introduction to biometric recognition", IEEE Trans. CSVT, vol. 14, no. 1, pp. 4-20, 2004.

2. J.J. Nora, F.C. Fraser, J. Bear, C.R. Greenberg, D. Patterson, D. Warburton, 'Twins and theirs use in genetics," in Medical Genetics: Principles and Practice $4^{\text {th }}$ ed, Philadelphia: Lea \& Febiger, 1994.

3. E.P. Richards, "Phenotype vs. genotype: why identical twins have different fingerprints" available at http://www.forensic-evidence.com/site/ID/ID_Twins.html

4. D. Cyranoski, "Detectors licked by gummy fingers" Nature, vol. 416, pp 676, 2002.

5. http://www.deleanvision.com/

6. Retinal technologies, http://www.retinaltech.com/technology.html

7. J. Daugman and C. Downing, "Epigenetic randomness, complexity and singularity of human iris patterns," Proceedings of the Royal Society, B, vol. 268, pp. 1737-1740, 2001.

8. A.W.K. Kong and D. Zhang, "Competitive coding scheme for palmprint verification," in Proc. ICPR, vol. 1, pp. 520-523, 2004. 
9. A.K. Jain, S. Prabhakar and S. Pankanti, "On the similarity of identical twin fingerprint," Pattern Recognition, vol. 35, no. 11 pp. 2653-2663, 2002.

10. R. Kimmel, Numerical Geometry of Image, Springer, New York, 2003

11. D. Voth, "Face recognition technology," IEEE Magazine on Intelligent Systems, vol. 18, no. 3, pp. 4-7, 2003

12. "Large scale evaluation of automatic speaker verification technology: dialogues spotlight technology report," The Centre for Communication Interface Research at The University of Edinburgh, May 2000, Available at http://www.nuance.com/assets/pdf/ccirexecsum.pdf.

13. K. Kodate, R. Inaba, E. Watanabe and T. Kamiya, "Facial recognition by a compact parallel optical correlator," Measurement Science and Technology, vol. 13, pp. 1756-1766, 2002.

14. C.C. Chibelushi, F. Deravi and J.S.D. Mason, "Adaptive classifier integration for robust pattern recognition," IEEE Trans. on SMC, Part B, vol. 29, no. 6, pp. 902-907, 1999

15. C. Simon and I. Goldstein, "A new scientific method of identification," New York state journal of medicine, vol. 35, no. 18, pp. 901-906, 1935.

16. P. Tower, "The fundus oculi in monozygotic twins: report of six pairs of identical twins," Archives of ophthalmology, vol. 54, pp. 225-239, 1955.

17. L. Flom and A. Safir, U.S. Patent No. 4641349, U.S. Government Printing Office, Washington, DC, 1987.

18. P.J. Phillips, A. Martin, C.L Wilson, M. Przybocki, “An introduction to evaluating biometric systems," Computer, vol. 33, no. 2, pp. 56-63, 2000.

19. Veinid, "http://www.veinid.com/product/faq.html"

20. D. Zhang, W.K. Kong, J. You and M. Wong, "On-line palmprint identification," IEEE Trans. PAMI, vol. 25, no. 9, pp. 1041-1050, 2003. 